\title{
OSTWALDS KLASSIKER \\ DER EXAKTEN WISSENSCHAFTEN
}

Begründet von Wilhelm Ostwald

NEUE FOLGE

Herausgegeben von

S. Balke, München; H. Gericke, München; W. Hartner, Frankfurt am Main; G. Kerstein, Hameln;

F. Klemm, München; A. Portmann, Basel; H. Schimank, Hamburg; K. Vogel, München

BAND 4 


\section{九童算街}

\section{CHIU CHANG SUAN SHU}

\section{NEUN BÜCHER ARITHMETISCHER TECHNIK}

Ein chinesisches Rechenbuch

für den praktischen Gebrauch aus der frühen Hanzeit (202 v. Chr. bis 9 n. Chr.)

Übersetzt und erläutert

von

\section{Kurt Vogel}

Forschungsinstitut des Deutschen Museums für die Geschichte der Naturwissenschaften und der Technik, München

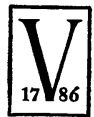


ISBN 978-3-322-97959-9 ISBN 978-3-322-98534-7 (eBook)

DOI $10.1007 / 978-3-322-98534-7$

1968

Alle Rechte vorbehalten

(C) 1968 by Springer Fachmedien Wiesbaden

Ursprünglich erschienen bei Friedr.Vieweg \& Sohn GmbH, Braunschweig 1968

Gesamtherstellung: G. Stalling AG, Oldenburg (Oldb.)

Umschlagentwurf: L. Nettelhorst, Wiesbaden

Best.-Nr. 9104 


\section{Vorwort}

Der bedeutende Beitrag der Chinesen zur Entwicklung der Mathematik ist im Abendland wenig bekannt geworden, obwohl vorzügliche zusammenfassende Darstellungen, vor allem die von Mikami [13], Needham [14(1)] und Juschkewitsch [9], leicht zugänglich sind. Es liegt wohl weniger an einem europazentrischen Standpunkt als daran, daß die grundlegenden Texte selbst, mit wenigen Ausnahmen, nur in chinesischer Sprache im Druck zur Ausgabe kamen. Diese Ausnahmen sind:

1. „Das mathematische Handbuch der Insel im Meer" von Liu Hui aus dem Jahre +263 [14 (1); 30], von L. van Hee ins Französische übersetzt [5];

2. „Das arithmetische Handbuch von Meister Sun“ von Sun Tzu aus der Mitte des 3. nachchristlichen Jahrhunderts, von È. I. Berezkina ins Russische übersetzt [2(3)];

3. „Neun Bücher arithmetischer Technik“ aus dem 1. vorchristlichen Jahrhundert, von È. I. Berezkina ins Russische übersetzt [2 (1)].

Gerade die letztgenannte Schrift, zu der das Handbuch von Liu Hui einen Nachtrag zum neunten Buch darstellt, ist ein Werk höchsten Ranges und in seinem Einfluß wohl das bedeutendste aller mathematischen chinesischen Bücher [14(1); 25]; es ist das älteste Lehrbuch der Rechentechnik überhaupt und mit seinen 246 Problemen als Aufgabensammlung ungleich reichhaltiger als andere aus der Antike, die sich in ägyptischen und babylonischen Texten erhalten haben. Griechische arithmetische Aufgabensammlungen (mit Ausnahme der bei „Heron“, die sich auf geometrische Probleme beschränken) kennen wir sogar erst aus späthellenistischer und byzantinischer Zeit. Erstmals sehen wir in den „Neun Büchern" neben Aufgaben der Unterhaltungsmathematik, die später Bestandteil aller mittelalterlicher Rechenbücher wurden, eine Matrizenrechnung zur Lösung linearer Gleichungssysteme, Regeln für das Rechnen mit allgemeinen Brüchen und negativen Zahlen. Wir sehen die dezimale Berechnung der Quadrat- und Kubikwurzel, Aufgaben der unbestimmten Analytik, die Behandlung von Problemen der Vermessungstechnik sowie bereits die Methode des doppelten falschen Ansatzes.

So erschien es nützlich, den Text selbst auch in einer anderen europäischen Sprache darzubieten und dabei einen Überblick über den in ihm aufgezeigten Wissensstand zu geben. 
Mein Dank gilt in erster Linie È. I. Berezkina, deren vorzügliche Bearbeitung mir als Ausgang zur Heranziehung des chinesischen Textes diente, dann all denen, bei denen ich philologische Hilfe erfahren durfte, Prof. H. Franke und W. Bauer sowie Dr. H. Hu ber vom Seminar für Ostasiatische Kultur- und Sprachwissenschaft der Universität München, Herrn Direktor Fr. J. Meier von der Ostasiatischen Abteilung der Münchner Staatsbibliothek und meinem Lehrer Pietro Liu, ferner Prof. H. Gericke vom Institut für Geschichte der Naturwissenschaften der Universität München, mit dem ich den mathematischen Inhalt eingehend diskutierte und dem ich manche Anregungen verdanke, sowie nicht zuletzt dem Verlag Friedr. Vieweg \& Sohn, der für eine einwandfreie Ausgabe und Ausstattung des neuen Ostwald-Klassiker-Bändchens besorgt war.

Kurt Vogel 


\section{Inhalt}

\section{Einleitung}

Der Text:

Neun Bücher arithmetischer Technik

(246 Aufgaben)

Buch I: Ausmessen von Feldern

1- 4 Das Rechteck ..................... 7

5-24 Regeln für das Rechnen mit Brüchen ....... 8

25-38 Die anderen ebenen Figuren ........... 12

Buch II: Regelung des Tausches von Feldfrüchten

1-31 Wertvergleich (Volumenvergleich) von Feldfrüchten ................... 17

32-37 Bestimmung des Einzelpreises aus Gesamtpreis und Menge gleichwertiger Gegenstände .......

38-46 Bestimmung des Einzelpreises, wenn die Gegenstände zwei verschiedene Preise haben (unbestimmte Probleme) .....................

Buch III: Proportionale Verteilung

1- 9 Gesellschaftsrechnungen .................

10-20 Schlußrechnungen (Regeldetri und Regula de quinque) $\ldots \ldots \ldots \ldots \ldots \ldots \ldots \ldots \ldots . . . \ldots$

Buch IV: Kleinere und größere Breite

1-11 Berechnung der Länge eines Rechtecks aus Fläche und Breite (mit Bruchrechnungen) ......... 35

12-16 Berechnung einer Quadratseite (Quadratwurzel). 39

17-18 Quadratwurzel beim Kreis .............. 41

19-22 Berechnung einer Würfelkante (Kubikwurzel) .. 41

23-24 Kubikwurzel bei der Kugel ............. 43

Buch V: Beurteilung der Arbeitsleistung

1-28 Inhaltsberechnung von Körpern; bei manchen Aufgaben, in denen es sich um die Herstellung von Wänden und Gräben handelt, auch Berechnung der Zahl der benötigten Arbeiter, wobei die jahreszeitlich verschiedene Leistung, der Transportweg und die Art des bearbeiteten Erdreiches zu berücksichtigen ist

Buch VI: Gerechte Steuereinschätzung

1- 4 Proportionale Verteilung einer Getreideabgabe oder einer Schanzarbeit auf die zur Ablieferung bzw. zur Abstellung verpflichteten Bezirke ..... 
5-28 Vermischte Probleme wie Bewegungsaufgaben, Mischungsrechnung, Zisternenproblem u.a. ....

Buch VII: Überschuß und Fehlbetrag

1-20 Vermischte Probleme, die mit dem ,doppelten falschen Ansatz" gelöst werden ........... 70

Buch VIII: Rechteckige Tabelle

1-18 Vermischte Probleme, bei denen lineare Gleichungssysteme bis zu 5 Unbekannten in einer Matrizenrechnung gelöst werden $\ldots \ldots \ldots \ldots .80$

Buch IX: Das rechtwinkelige Dreieck

1-16 Angewandte Beispiele zum Pythagoreischen Lehrsatz ............................ 90

17-24 Vermessungsaufgaben ................ 98 Der mathematische Inhalt

Die Zahlen und ihre Wiedergabe ........... 105

Die Brüche ....................... 107

Die Grundrechnungen ................ 108

Quadrat- und Kubikwurzel .............. 113

Weitere Kenntnisse aus Arithmetik und Algebra 120

Geometrische Kenntnisse ................ 122

Die Aufgaben ............................ 124

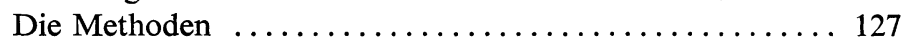

Anhang: Die Maße .......................... 139

Chinesische Wörter (insbesondere der mathematischen Fachsprache) ............... 142

Literaturverzeichnis ........................ 150

Namen- und Sachregister ..................... 154

Schrifttafel ............................ 159 